\title{
Epidemics with a Path to Quantum Epidemics
}

\author{
Omar Alzeley * \\ Department of Mathematics, University of Leicester, United Kingdom \\ *Corresponding author: Omar Alzeley , Department of Mathematics, University of Leicester, University Road, Leicester LE1 7RH, \\ United Kingdom
}

To Cite This Article: Omar Alzeley. Epidemics with a Path to Quantum Epidemics. Am J Biomed Sci \& Res. 2019 - 5(5). AJBSR.MS.ID.000941. DOI: 10.34297/AJBSR.2019.05.000944.

Received: 眥 September 30, 2019; Published: 眥 October 11, 2019

\begin{abstract}
Classical epidemics are based on classical probability. In this paper, we review classical epidemics, the generalization of which to quantum probability is fundamentally interesting

Keywords: epidemic, quantum probability, stochastic models
\end{abstract}

\section{Introduction}

SIR is an effective means of analysing a standard infection scenario, whereby (S) stands for the susceptible people who are healthy but can contract the disease, infectives (I) accounts for those who have contracted and can transmit a disease, and the removed cases (R) accounts for a population that leaves the infection either through death or recovery [5]. Investigated the SIR model using the analytical matrix method in which they determined the distribution of the final stage of the process. Epidemic models evaluate the manner in which infectious diseases spread in a population. They also made several interesting measures of the dimensions of the epidemic. However, the model has not been investigated in terms of attaining threshold data for reference and validity [1] presents a case for SIS and SIR epidemic models for individual populations and insists that the SIS model has only been applied to sexually transmitted diseases. However, SIS fails to account for the spread of some epidemics because it makes the assumptions that there are no vertical transmissions and no disease-related deaths. SIR is a bivariate process with joint probability functions for random variables, unlike the SIS model.

Early models such as the Kermack-McKendrick model for assessing infection use typical mathematical estimations that assess the difference between a disease-free equilibrium and the approach to an endemic equilibrium [2]. However, the early Kermack-McKendrick model failed to assess arbitrary distributions. Consequently, stochastic models replaced the simple model because once infective individuals transmit an infection this becomes a stochastic event that depends on the patterns of contact between persons within a population [2]. Likewise, [3] explain that epidemic modelling for diseases like malaria and HIV follow the progress of the outbreak and mechanisms of infection, which present a deterministic model within a stochastic setting. The analysis is either deterministic or stochastic and happens either in continuous or discreet time [5] investigated epidemic using a continuous-time stochastic approach. They applied the general epidemic process and used the Markovian formula for infinitesimal transition probabilities [4] explains that the special Markovian case explains a situation in which the infectious period is defined by an exponential distribution known as the general stochastic epidemic. One of the conditions required for a general stochastic epidemic is the existence of a typical infected individual within a susceptible but homogenous population [4]. The use of a Markov process is based on a block-structured generator that responds to the number of susceptible or infective individuals, or those removed from the infection phases [5] applied the block-structured Markovian process to evaluate and treat an SIR-type epidemic. The model is guided by three aspects: the first is that the phase process records a specific number of infectives with other infection sources. The second asserts that transitions take place and are phase-type instead of exponential in nature. The last claims that contamination is modelled in a general manner because infection happens arbitrarily.

[5] Insist that the final state of an epidemic terminates when the infection dies after undergoing transitions without producing any new infections, as based on the extended general epidemic 
theory. Detection is achieved through the Poisson process with successive detection times. If an infection is successfully killed when detected, then the infinitesimal transition probability claim that the epidemic terminates after some time. However, if the infective agents exhibit several strains that complicate the control mechanisms. Transmission of two strains is easier to deal with because the infected person only contains a specific pathogen obtained from the infected person, which requires computation of the individual strains. However, the SRI method lacks a mechanism of assessing the on-set and termination of an epidemic with several strains that counter-interact [6] found that the severity of an epidemic outbreak is directly correlated with the structure of the population. The study develops a stochastic SIR household epidemic model whereby individuals within a household are likely to be highly infected due to the consistency of their interactions compared to those with other members of the population. The model is effective in giving a precise dynamic view of transmissions compared to the traditional model where all individuals within a population are considered to represent a mix of a homogenous group. The use of SIR with a closed population in a household model mimics the urban lifestyle where people live together without showing a high rate of migration. Likewise, splitting the population into small households is effective in understanding the ways in which epidemics spread based on the SIR model, whereby all members are susceptible to every disease introduced because of contacting an infective individual. Consequently, it is reasonable to assume that all members of a household have a similar probability of contracting a disease and accordingly predict the future course of an outbreak

Various studies have used a deterministic, discreet-time model to assess the phases of an epidemic in which researchers divide a community into smaller populations composed of individual households. By contrast, a stochastic SIR model provides a different notion about the epidemic. The objective of the SIR model is to estimate data about an infection as derived from a small and localized place and the use of established parameters to estimate the maximum possible effects of a disease in a large population. Outlaying an infection rate requires a consistent study of the behaviour of the emerging epidemic to find parameters that allow the estimation of the termination phase. Knowledge of the dynamics is critical to controlling diseases and combating them before they spread to other populations. However, a deterministic, discreettime model fails to meet the real-time growth rate of an emerging epidemic. A relationship exists between the real-time growth rate and the reproduction numbers of an emerging epidemic. However, to date, very little of value has been achieved in terms of estimating data for an emerging phase of an epidemic. Likewise, the frequency of contact even between household members determines the rate of infection. Government and other health institutions are concerned about the means of combating emerging epidemics.
Vaccinations deal with outbreaks as a preventive measure that reduces the parameters to infectives in the sense of reducing the possibility of a large-scale outbreak. Therefore, immunization reduces the anticipated size of a stochastic discreet-time epidemic. However, vaccines have potential problems because vaccination within a stochastic epidemic model has the intention of reducing the branching process but may fail to achieve the desired effect. An alternative way of handling immunization is by giving vaccines to a limited number of people within a population to avoid making a large group of people ill with a vaccine that they do not need. Immunization faces challenges that hinder the anticipated positive effects that include the facts that, sometimes, a vaccine will fail to work and that there is always the possibility of a new strain of a disease developing that counters or is otherwise unaffected by the vaccine. These two problems demand that immunization should focus on covering only a minimum number of a population to counter an outbreak. Likewise, the study found that under a deterministic model, under-vaccination occurs if one is dealing with a heterogeneous population that tends to mix randomly.

Application of the Bayesian Markov Chain Monte Carlo (MCMC) approach provides an effective method of combating real-life epidemics using datasets based on the SIR household epidemic model with a finite population [4] asserts that the availability of computing machines has made the MCMC algorithm accessible for computing complex Bayesian models. A researcher can use MCMC to create approximate samples of a posterior distribution by generating a Markov chain with a posterior and its limiting distribution [6] insists that the covariance matrices can investigate the values of sampled households that represent the entire population. Moreover, estimates depend on the assumption that all observed households have a mutually independent outcome, which means that only infected households are integrated into the research [6] cautions against using several datasets from different outbreaks to determine the rate of an epidemic using the household SIR model because once the sample is too large, the imposing household size interferes with the final probabilities and covariance matrices [6] compares the results of an investigation of the household population using the basic model in contact is independent of household size, the Cauchemez mode, in which local contact rate varies with household size, and the unrestricted model, where local contact rates with households of different sizes are independent of each other. The basic and Cauchemez models provide the best fit for observed data so long as infection rates are minimal within a population. The study concluded that the Bayesian method MCMC provides a credible calculation for intervals for standard error. [4] asserts that when a stochastic epidemic affects a homogeneous population, it is generalized with a specific exponential distribution that follows the Markovian model. SIR's Cauchemez models are effective in determining the contact rate at the household level and for understanding the effects of the 
post-vaccination epidemic model. If the increase in the local contact parameters of an epidemic are very large, then single household epidemics infect everyone not immune, which makes no difference when adopting either a basic or Cauchemez model. However, an exception is possible if the population under consideration has a large vaccination coverage.

\section{Conclusion}

Previous work has focussed purely on classical probability tools to investigated epidemics; few researchers have addressed the quantum probability tool. Therefore, generalizing to quantum probability is fundamentally interesting. Investigating the SIS and SIR model by making use of the quantum random walk is one possibility for future work. Also, studying SIS using the analytic matrix method should also be an interesting topic for consideration.

\section{Acknowledgements}

The author is grateful to Professor Sergey Utev (Department of Mathematics, University of Leicester), for introducing him to the subject of quantum probability. Most of my initial interest in mathematical epidemiology is a result of the patient explanations given by Professor Sergey.

\section{References}

1. Allen LJ (2010) An introduction to stochastic processes with applications to biology. Chapman and Hall/CRC.

2. Brauer F (2017) Mathematical epidemiology: Past, present, and future. Infectious Disease Modelling 2(2): 113-127.

3. Daley DJ, Gani J (2001) Epidemic modelling: an introduction. Cambridge University Press, USA.

4. Demiris N (2004) Bayesian Inference for stochastic epidemic models using Markov chain Monte Carlo methods. PhD thesis, University of Nottingham, England.

5. Lefe vre C, Simon M (2019) Sir-type epidemic models as blockstructured markov processes. Methodology and Computing in Applied Probability, pp. 1-21.

6. Shaw LM (2016) SIR epidemics in a population of households. PhD thesis, University of Nottingham, England. 